\title{
DE ROSTROS A ESPACIOS COMPOSITIVOS: UNA PROPUESTA ESTILÍSTICA PARA EL VALLE DE CHALINGA, CHILE*
}

\author{
FROM FACES TO COMPOSITIONAL SPACE: A STYLISTIC \\ PROPOSAL FOR THE CHALINGA VALLEY, CHILE
}

\author{
Gloria Cabello ${ }^{1}$
}

\begin{abstract}
Presentamos un estudio del arte rupestre del valle de Chalinga a través del análisis detallado de motivos análogos a las conocidas máscaras del Estilo Limarí. Cambiando el sugestivo concepto de "máscara" por el de "representaciones a partir de un marco", consideramos los motivos como un espacio compositivo donde los elementos se combinan a partir de reglas de diseño. Estas combinatorias son observadas desde la simetría y la estadística, para proponer una secuencia de diseño, que -cotejada con data arqueológica independiente- nos permite discutir el desarrollo del arte rupestre de Chalinga frente al estilo Limarí y su autoría en relación con las poblaciones alfarero tempranas y diaguitas presentes en el área.
\end{abstract}

Palabras claves: estilo, motivo, representaciones a partir de un marco, campo compositivo, secuencia de diseño.

This article presents a study of rock art from the Chalinga valley in Chile focusing on a set of motifs similar to the famous masks of the Limarí style. Moving from the suggestive concept of "mask" to that of "framed figures", we consider the rock art motifs as compositional spaces within which elements are combined according to specific rules of design. These combinations are studied both statistically and in terms of symmetry in order to propose a design sequence, which -in conjunction with other independent archaeological data-allow us to discuss the development of Chalinga's rock art vis-a-vis the Limari style and its relation with the populations from Early Ceramic and Diaguita Periods present in this area.

Key words: Style, motif, framed figures, compositional space, design sequence.

Hasta los años noventa, la prehistoria del Choapa se proyectaba según la arqueología de otras zonas del norte semiárido. El arte rupestre no era excepción, incluyéndose por extensión en el estilo Limarí, que Mostny y Niemeyer (1983) definieron a partir del sitio El Encanto y atribuyeron a la también hasta entonces homogénea cultura El Molle (0-800 d.C.).

El estilo Limarí agrupa una gran variedad de motivos, siendo características las "máscaras": rostros cuadrangulares o circulares que presentan distintos tipos de tocado, como apéndices espiralados, apéndices laterales hacia arriba o tocado semicircular decorado al interior; estas últimas también conocidas como cabezas-tiara (Mostny y Niemeyer 1983) (Figura 1).

Revisiones posteriores de Castillo (1985 y 1990) objetan la pertinencia del estilo Limarí y su limitada adscripción a El Molle. Similitudes formales de algunos de estos motivos con elementos de la decoración cerámica diaguita e incluso inca han sido mencionadas por Iribarren (1973),
Jackson et al. (2002) y Castelleti (2004). Troncoso (2004) profundiza sobre estas semejanzas formales y visualiza, en la disposición de los motivos en el soporte, estructuras organizacionales que serían propias de cada período ${ }^{1}$.

Intentando avanzar en el problema estilístico del arte rupestre del Choapa y su adscripción cronocultural, nos propusimos retomar la discusión sobre los motivos tipo máscaras Limarí que se encuentran en el valle de Chalinga (Cabello 2003). Esta vez los analizamos a partir de su diseño y organización dentro del panel, para luego relacionarlos con información contextual y compararlos con otros referentes visuales de las culturas evidenciadas en el área (Cabello 2005)².

\section{EI Escenario}

El río Chalinga nace en la alta cordillera de la confluencia del río de Los Helados con el estero de Las Puentecillas y desemboca en el río Choapa, cerca de Salamanca (Figura 2). Restos arqueológicos

\footnotetext{
* Este estudio fue presentado en el simposio sobre Arte Rupestre de los Andes, realizado en el $52^{\circ}$ Congreso Internacional de Americanistas, Sevilla, julio de 2006. La edición de los artículos seleccionados para este número de la Revista estuvo a cargo de Marcela Sepúlveda y Jean Guffroy.

1 Centro de Investigaciones del Hombre en el Desierto, Universidad de Tarapacá, Arica, Chile. glcabello@ gmail.com
} 


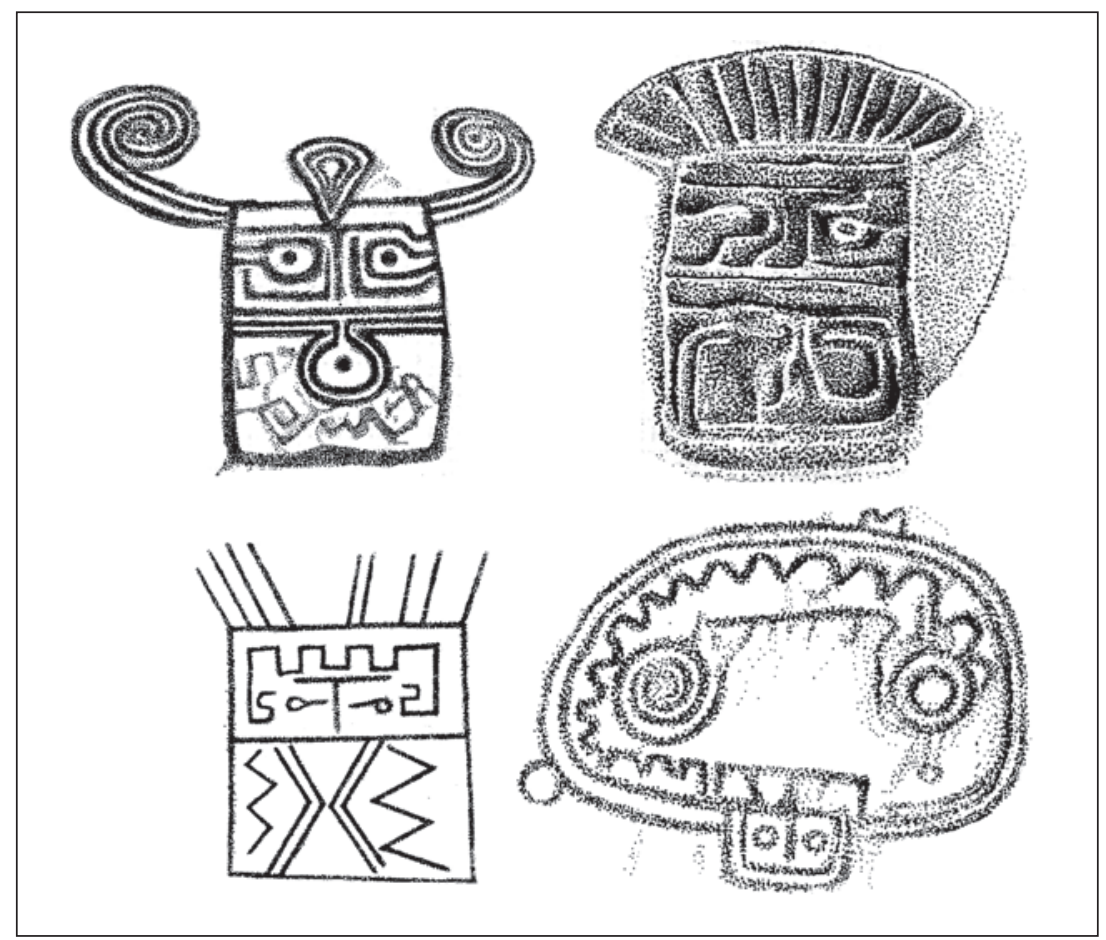

Figura 1. Mascariformes del estilo Limarí (tomado de Mostny y Niemeyer 1983). Masks of Limarí style (after Mostny and Niemeyer 1983).

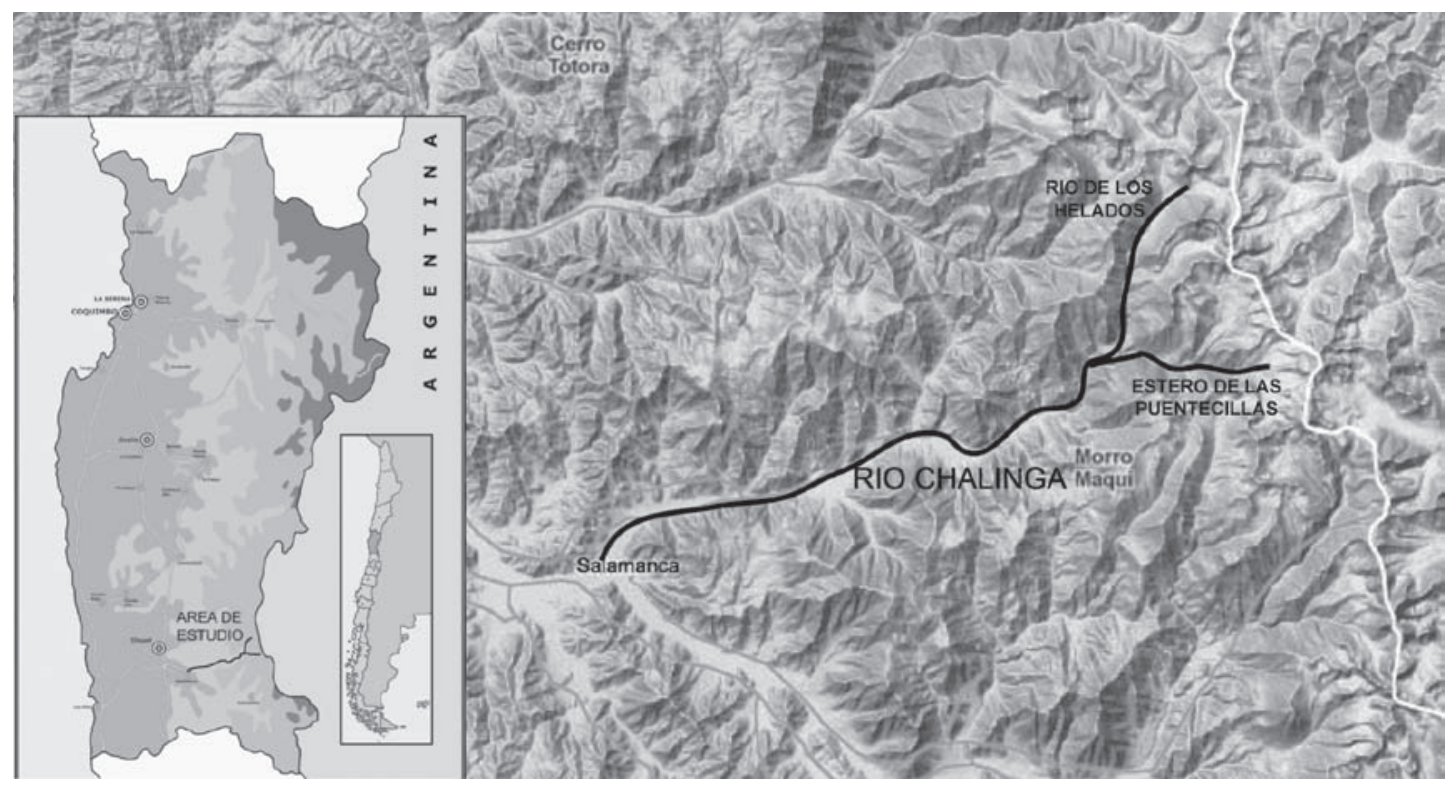

Figura 2. Mapa con la ubicación del valle de Chalinga.

Map showing location of the Chalinga Valley. 
identificados (Becker 2003) señalan que el valle habría estado ocupado intensamente desde el período Alfarero Temprano, por grupos que presentan rasgos cerámicos propios y locales, que se alejan de lo definido como Molle ${ }^{3}$. Esta tradición alfarera (PAT en adelante) estaría perdurando en algunos sectores del Choapa hasta el 1500 d.C. (Pavlovic 2004; Pavlovic y Rodríguez 2005; Urízar 2004), siendo contemporánea a los diaguita e incluso al Inka. En Chalinga, el registro de 120 sitios PAT frente a siete Diaguita, se explica por condiciones geográficas y climáticas que favorecen el modo de vida cazador recolector/hortícola del PAT, mientras resultan impropias a la economía agrícola diaguita (Pavlovic y Rodríguez 2005). Los diaguita, entonces, habrían ocupado el valle de forma marginal y durante momentos más bien tardíos, como lo evidencian los dos sitios de mayor relevancia (R5 y LEA).

Por otra parte, un total de 76 sitios de petroglifos fueron detectados y registrados a lo largo del valle ${ }^{4}$. En 20 de ellos se reconocieron máscaras tipo Limarí ( $n=61$ ), así como otros motivos similares que no se ajustan a la definición dada por Mostny y Niemeyer (1983). Es por ello que decidimos dejar la búsqueda de referentes figurativos que conlleva el concepto "máscara" y reemplazarlo por "representaciones dentro de un marco", lo que nos permitió no sólo ampliar la muestra a 82 , sino que ver el motivo como un campo de composición donde los elementos se combinan de una forma particular para darle unidad visual a los diseños (Gallardo 2009).

\section{El Análisis}

Coincidimos con Davis (1990) en que el estilo no está en el material, sino que debe ser descubierto y escrito por alguien. Para este autor, estilo es la descripción de un conjunto politético de atributos similares pero variables presentes en un grupo de artefactos, cuya presencia se explica mediante la historia de ellos y, especialmente, por la descendencia común de un sistema de producción que puede identificarse arqueológicamente (Davis 1990).

Para hacer esta descripción, Davis (1990) propone que el conjunto de atributos debe ser elegido por el investigador, pero la distancia y la causa de relación entre dos atributos deben establecerse mediante métodos independientes, p.ej., estadísticos.

En nuestro caso, el procedimiento seguido fue un Tree clustering ${ }^{5}$ para 82 representaciones dentro de un marco, definiendo como atributos: marco (circular/ rectangular), segmentación horizontal (presencia/ ausencia), elementos del segmento superior e inferior (rectilíneo/ondulado/ausente), evocación a rostro (sí/no) y ejecución de movimientos de simetría en el diseño general y en las unidades fundamentales (sensu Washburn y Crowe 1988) (Figura 3).

Las variables fueron sometidas a tres análisis estadísticos de agrupamiento, que contrastados generaron siete tipos de motivos (I a VII), distintos en los elementos utilizados y su organización dentro del marco, independientemente que la forma de éste sea circular o rectangular.

Teniendo el marco como campo de composición básico, el tipo I ( $\mathrm{n}=22$, Figura 4) presenta elementos ondulados que hacen clara referencia al rostro y que se disponen simétricamente a partir de un eje vertical imaginario. El tipo II ( $n=8$, Figura 5), en cambio, presenta elementos abstractos que se disponen libremente al interior del marco.

En un campo de composición segmentado mediante un trazo horizontal, pero siguiendo la misma organización anterior, el tipo III ( $\mathrm{n}=13$, Figura 6) es figurativo simétrico y el IV ( $n=4$, Figura 7), abstracto asimétrico.

Cuando el marco dividido actúa como dos campos de composión independientes (arriba figuras geométricas que evocan rostros se disponen simétricamente y abajo los elementos siguen otros movimientos de simetría) tenemos al tipo $\mathrm{V}(\mathrm{n}=14$, Figura 8) con elementos ondulados que se trasladan; y en el VI ( $n=15$, Figura 9), rectilíneos que se trasladan y reflejan especularmente en distintos ejes, llegando a veces a formar diseños discretos y simétricos.

En el tipo VII (n=6, Figura 10), una línea vertical explicita el eje simétrico del diseño general. Esto genera un campo de composición cuatripartito, donde se replica el mismo principio simétrico -y a veces la misma unidad fundamental- en todos los segmentos, obteniéndose patrones bidimensionales. Con ello el campo de composición se convierte en un lugar donde se ejercitan movimientos de simetría, separándose completamente de la evocación del rostro ${ }^{6}$.

Para identificar sistemas de producción comunes recurrimos a los criterios asociativos propuestos por Gallardo (1996): contraste, contigüidad y semejan$\mathrm{za}^{7}$. Las dos primeras fueron evaluadas cotejando la tipología propuesta con el tipo de sitio y su adscripción cronocultural ${ }^{8}$ (Becker 2003) (Tabla 1), 


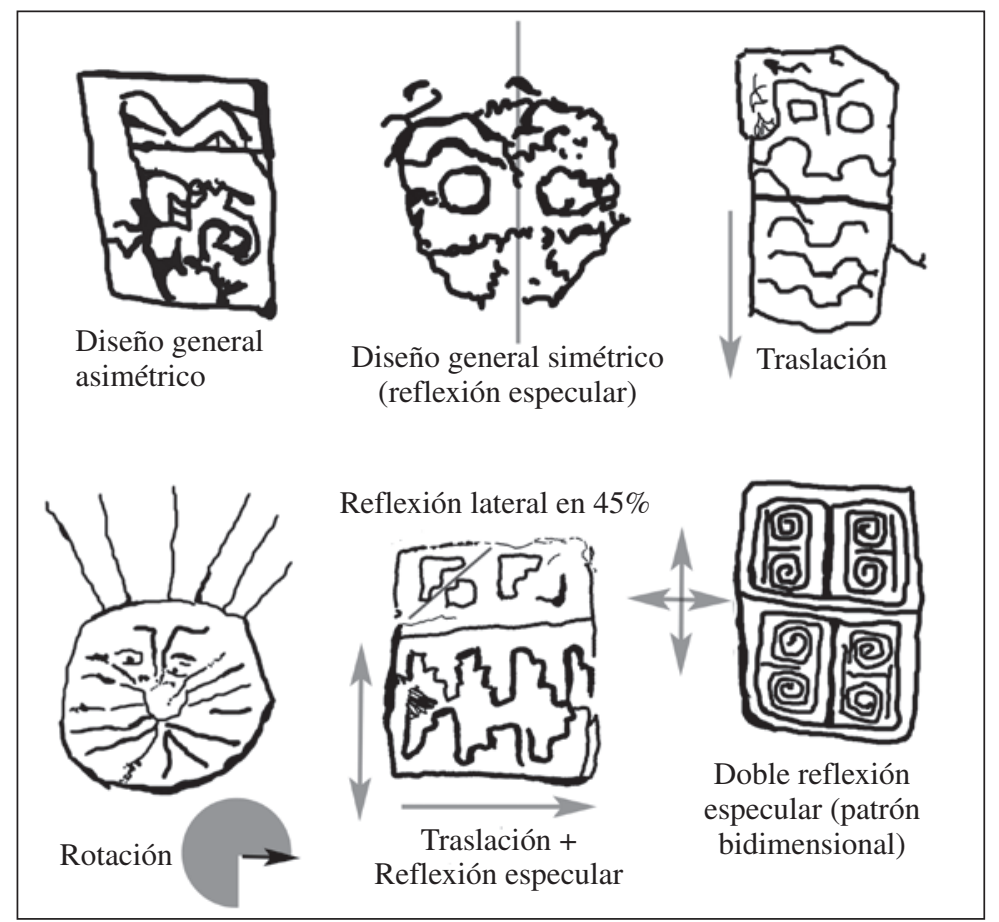

Figura 3. Ejemplos de movimientos de simetría en las figuras a partir de un contorno.

Examples of symmetry movements in the framed motifs.
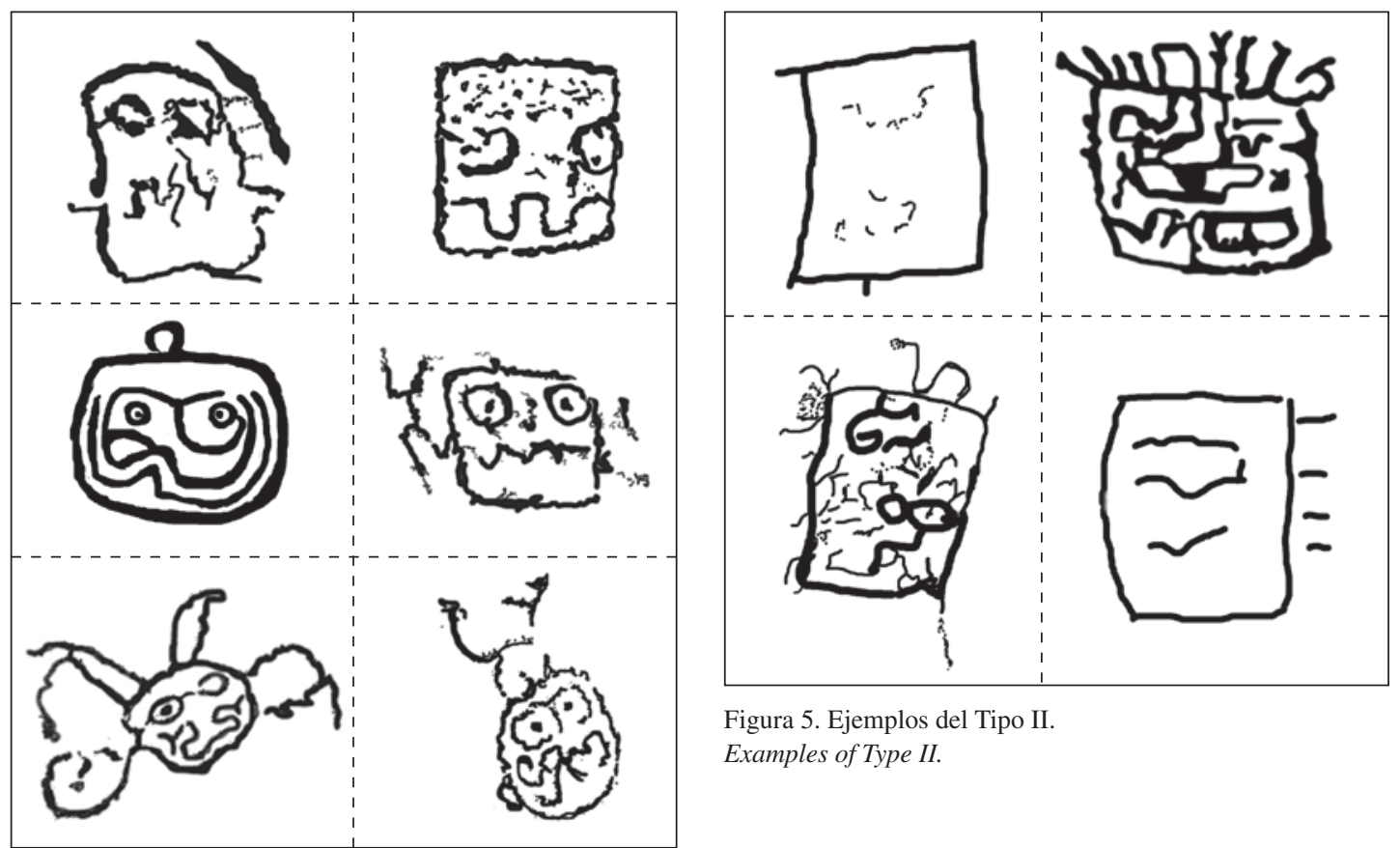

Figura 5. Ejemplos del Tipo II.

Examples of Type II.

Figura 4. Ejemplos del Tipo I.

Examples of Type I. 


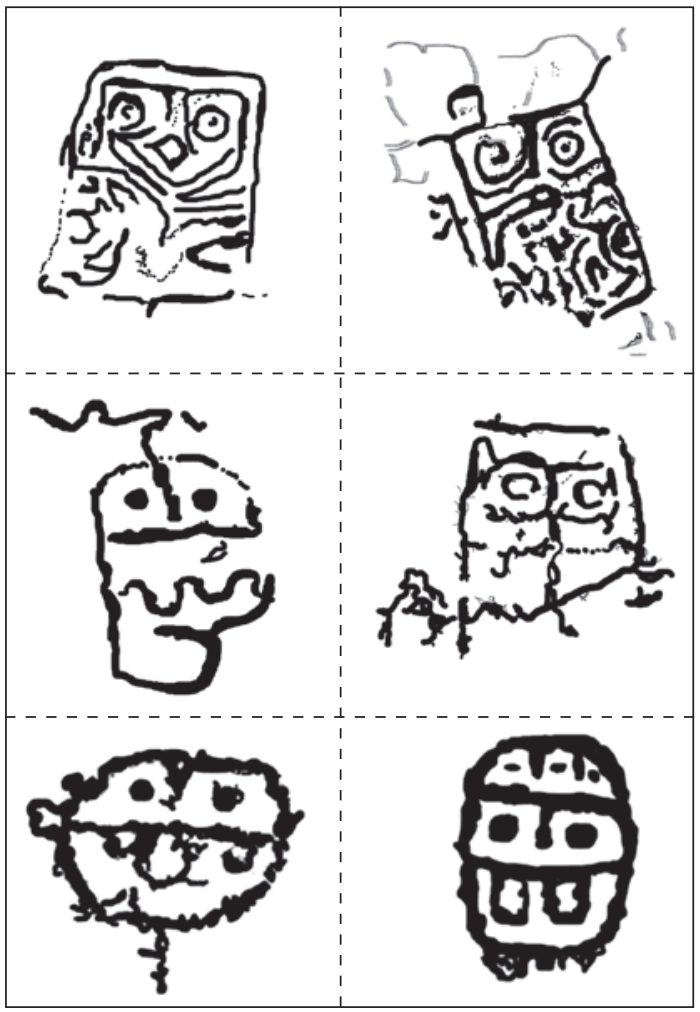

Figura 6. Ejemplos del Tipo III.

Examples of Type III.

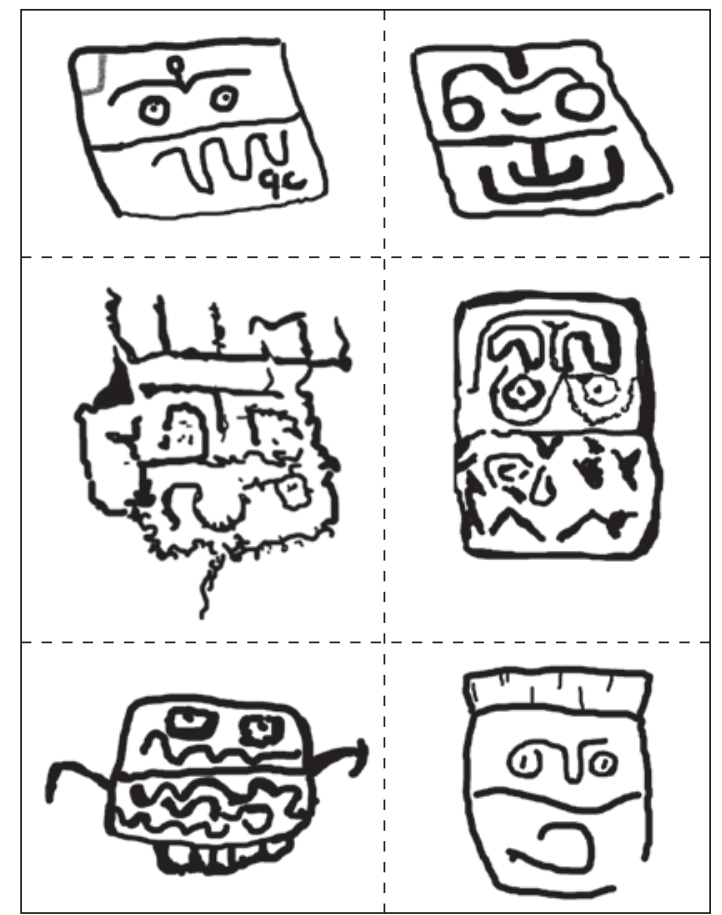

Figura 8. Ejemplos del Tipo V.

Examples of Type $V$.

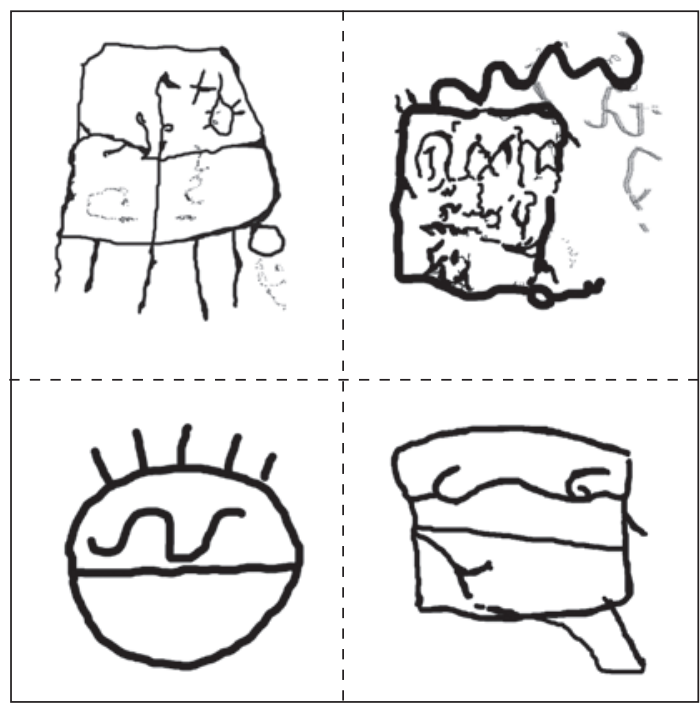

Figura 7. Ejemplos del Tipo IV.

Examples of Type IV.

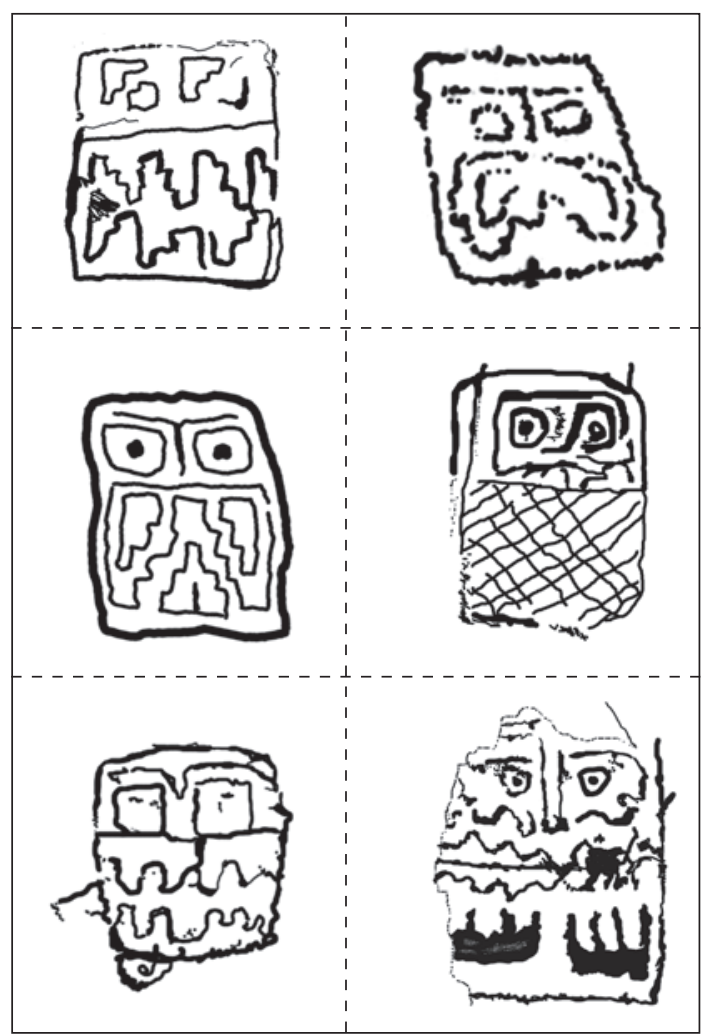

Figura 9. Ejemplos del Tipo VI.

Examples of Type VI. 


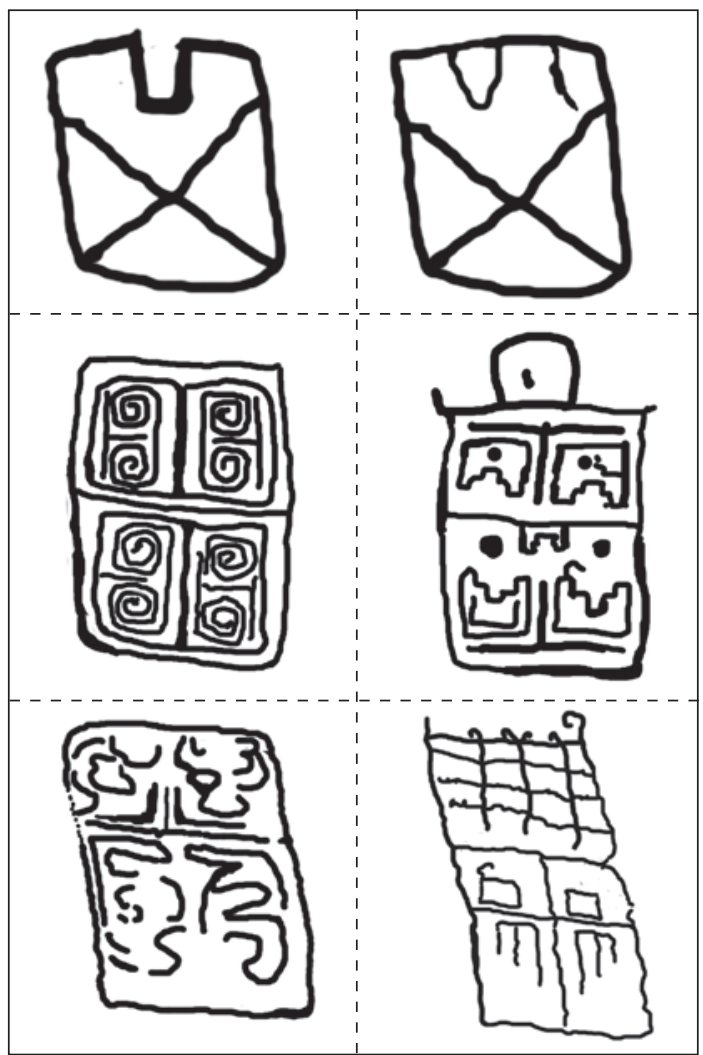

Figura 10. Ejemplos del Tipo VII.

Examples of Type VII. observándose dos modalidades diferentes. La primera corresponde a sitios que pueden presentar cerámica PAT, ubicados en terrazas fluviales, con bloques de tamaño mediano que presentan una amplia variedad de motivos, entre los que se encuentran representaciones dentro de un marco de los tipos I, II, III o IV. Generalmente encontramos una de estas figuras en el centro del panel, dispuesta junto a figuras abstractas (líneas rectas o curvas sin referente conocido) en baja cantidad (uno a cuatro) y comparativamente pequeñas (dos o tres veces menor), lo que hace que las representaciones dentro de un marco adquieran protagonismo (Figura 11).

La segunda modalidad son sitios que pueden presentar cerámica PAT y diaguita, ubicados en laderas de cerro o pie de monte, de grandes dimensiones, con numerosos bloques medianos y pequeños dispersos o bien uno de gran tamaño (más de 6x6 m). En estos sitios encontramos representaciones dentro de un marco de los tipos $\mathrm{V}$ a VII, pero no de forma exclusiva, sino que pueden hallarse también los tipos anteriores. De hecho, estas figuras tienden a aumentar su representación en relación con los otros motivos del sitio y/o del panel. Las representaciones a partir de un marco allí encontradas son de tamaño similar o levemente superior a la gran cantidad y variedad de motivos

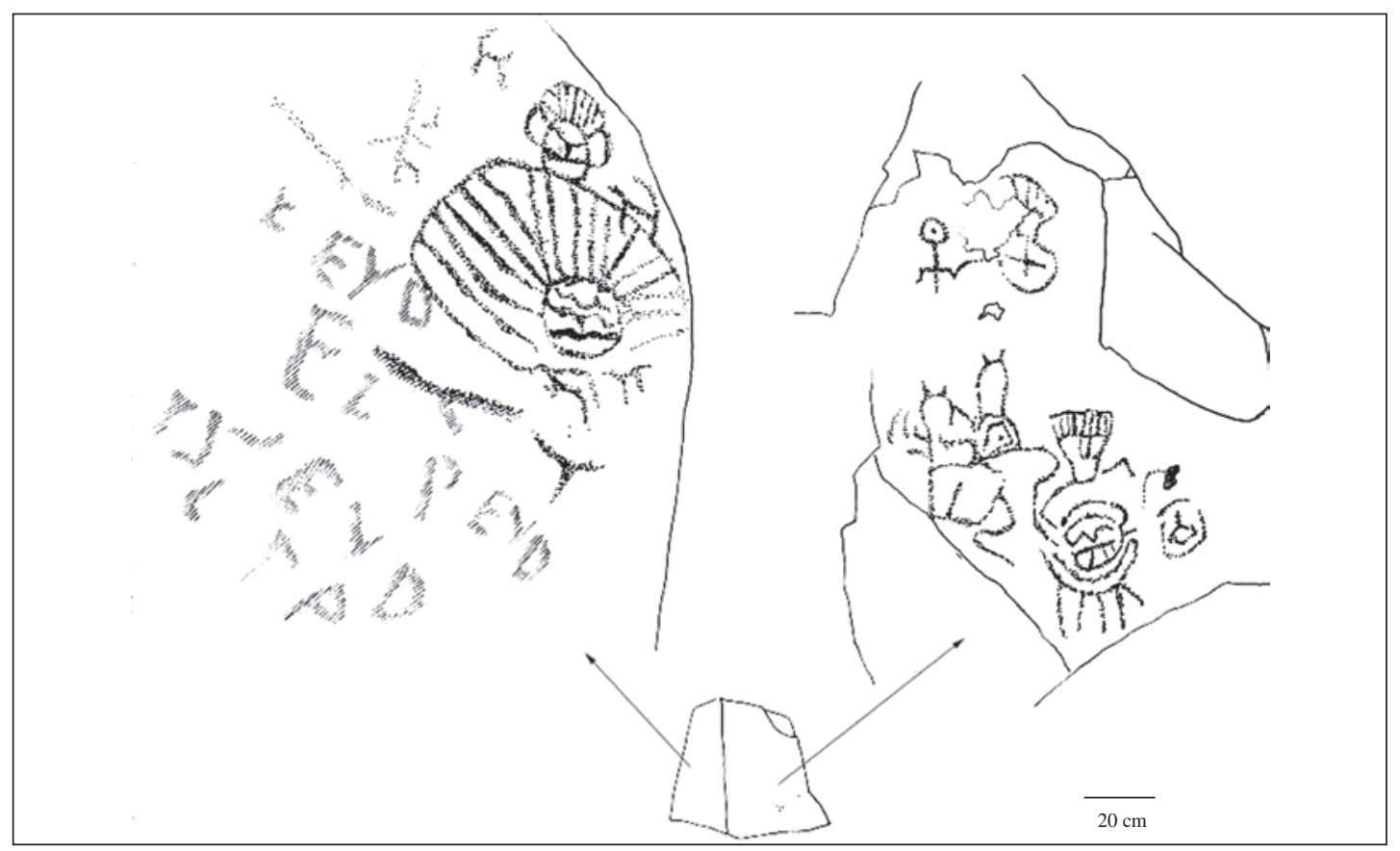

Figura 11. Ejemplo de panel de la primera modalidad (CH01) (tomado de Artigas 2003).

Example of a panel exhibiting the first modality (CHO1) (after Artigas 2003). 
Tabla 1. Presencia de los Tipos por sitio arqueológico, indicando su filiación cronocultural y fechados absolutos cuando los tienen (tomado de Becker 2003).

Presence of types by archaeological site, indicating their chrono-cultural affiliation and absolute dates, where available (after Becker 2003).

\begin{tabular}{|c|c|c|c|c|c|c|c|c|c|}
\hline \multirow{2}{*}{ Sitio } & \multirow{2}{*}{ Filiación } & \multirow{2}{*}{ Fechas } & \multicolumn{7}{|c|}{ Tipo } \\
\hline & & & $\mathrm{I}$ & II & III & IV & V & VI & VII \\
\hline Z06 & Indeterminada & & & & & & & $\mathrm{X}$ & \\
\hline Z09 & Indeterminada & & $\mathrm{X}$ & & & & & & \\
\hline Z11 & Indeterminada & & $\mathrm{X}$ & & & & & & $\mathrm{X}$ \\
\hline $\mathrm{Z} 12$ & Alfarero Temprano & & $\mathrm{X}$ & $\mathrm{X}$ & & & & $\mathrm{X}$ & \\
\hline $\mathrm{Z} 13$ & Alfarero Temprano & & & $\mathrm{X}$ & & & & & \\
\hline T01 & Alfarero Temprano y Diaguita & $\begin{array}{l}\text { 1090 } \pm 90 \text { d.C. (UCTL 1397) } \\
\text { Superficie }\end{array}$ & & & & & $\mathrm{X}$ & & \\
\hline T02 & Alfarero Temprano & 1170 \pm 80 d.C. (UCTL 1398) Superficie & $\mathrm{X}$ & $\mathrm{X}$ & $\mathrm{X}$ & $\mathrm{X}$ & $\mathrm{X}$ & $\mathrm{X}$ & \\
\hline R01 & Alfarero Temprano & $\begin{array}{l}855 \pm 100 \text { d.C. }(\text { UCTL } 1341) \\
\text { Pozo1 Nivel } 4(30-40 \mathrm{~cm}) \\
1420 \pm 60 \text { d.C. (UCTL } 1447) \\
\text { Pozo5 Nivel } 2(10-20 \mathrm{~cm})\end{array}$ & $\mathrm{X}$ & $\mathrm{X}$ & $\mathrm{X}$ & & & & \\
\hline SA06 & Alfarero Temprano & & & & $\mathrm{X}$ & & & & \\
\hline SA07 & Alfarero Temprano & & $\mathrm{X}$ & & $\mathrm{X}$ & & $\mathrm{X}$ & $\mathrm{X}$ & \\
\hline SA17 & Alfarero Temprano & & $\mathrm{X}$ & & $\mathrm{X}$ & $\mathrm{X}$ & $\mathrm{X}$ & $\mathrm{X}$ & $\mathrm{X}$ \\
\hline SA19 & Alfarero Temprano y Diaguita & & $\mathrm{X}$ & $\mathrm{X}$ & & $\mathrm{X}$ & $\mathrm{X}$ & $\mathrm{X}$ & \\
\hline SA20 & Alfarero Temprano & & & & $\mathrm{X}$ & & & & \\
\hline SA21 & Indeterminada & & $\mathrm{X}$ & & & & & & \\
\hline CU01 & Alfarero Temprano y Diaguita & & $\mathrm{X}$ & $\mathrm{X}$ & & & & & $\mathrm{X}$ \\
\hline CU02 & Indeterminada & & & & $\mathrm{X}$ & & & & \\
\hline CU03 & Diaguita & & & & $\mathrm{X}$ & & $\mathrm{X}$ & & $\mathrm{X}$ \\
\hline $\mathrm{CH} 01$ & Indeterminada & & $\mathrm{X}$ & & & & & & \\
\hline $\mathrm{CH} 02$ & Indeterminada & & & $\mathrm{X}$ & & & $\mathrm{X}$ & & \\
\hline EP01 & Indeterminada & & & & & & & $\mathrm{X}$ & \\
\hline
\end{tabular}

presentes, por lo que no destacan al interior del panel. En ellos se disponen figuras geométricas abundantes (principalmente variaciones del círculo) y figurativos (antropomorfos y zoomorfos). Estos pueden alcanzar cierto grado de complejidad, ya sea en la confección del diseño (cuerpos realizados mediante un contorno que se rellena de puntos) o en su composición (constituir escenas, p.ej., antropomorfo guiando a camélido) (Figura 12).

Consideramos necesario remarcar que ambas modalidades parecen ser opciones netamente de orden visual, sin observarse diferencias en la técnica de ejecución o en las pátinas de motivos de un mismo panel que nos hagan separar práctica y temporalmente a los autores ${ }^{9}$. Sólo en uno de los sitios de la segunda modalidad (Figura 12) se distingue una tendencia a la horizontalidad y oblicuidad de los motivos en el panel, siguiendo lo propuesto por Troncoso (2004). A nuestro juicio prima más bien una distribución dispersa, que sin embargo aprovecha la forma y el tamaño del soporte para resaltar la expresión visual de los motivos.

Atendiendo a la búsqueda de semejanzas (sensu Gallardo 1996), nos centramos en referentes visuales PAT y diaguita que fueran estructuralmente comparables a las representaciones dentro de un marco: las bandas de decoración de las vasijas. Ellas son campos de composición en cuyo interior se aplican principios simétricos particulares que generan diseños y patrones decorativos distintivos para cada grupo cultural analizado. En vasijas del PAT, de decoración incisa y heterogénea, se observan unidades mínimas simples -como triángulos o líneas- que utilizan la traslación y reflexión 


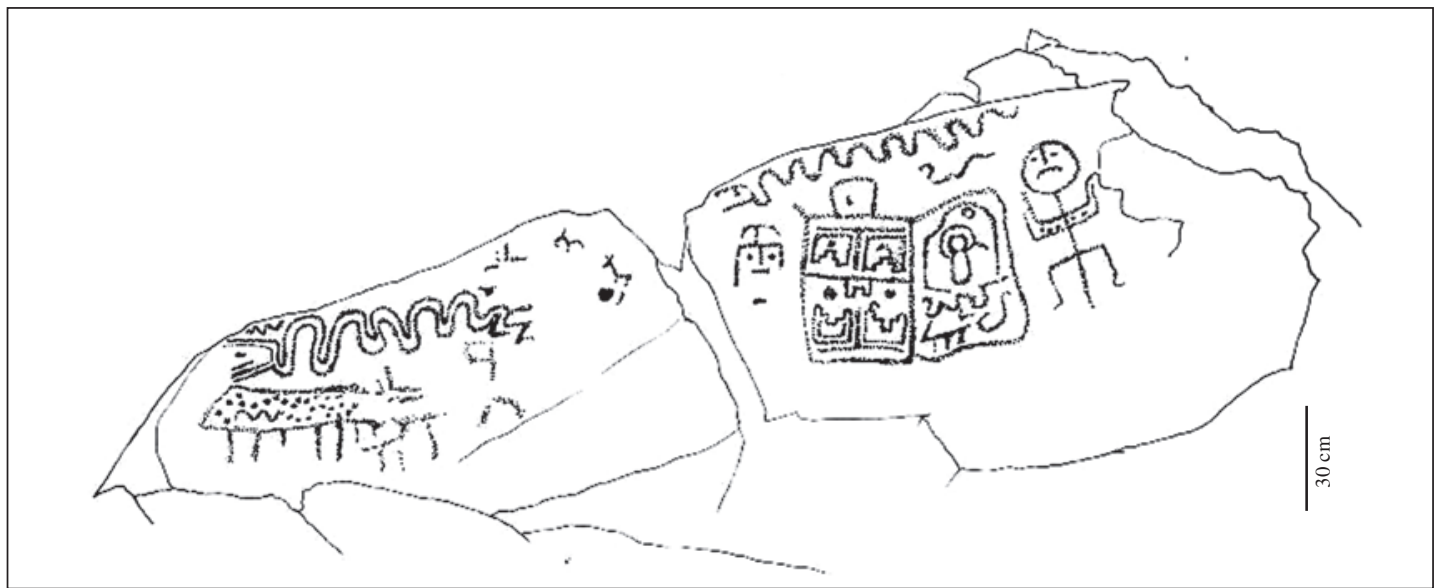

Figura 12. Ejemplo de panel de la segunda modalidad (CU01) (Tomado de Artigas 2003).

Example of a panel exhibiting the second modality (CU01) (after Artigas 2003).

especular para generar estructuras simétricas también simples, como el chevrón (González 1995, 1997; Pavlovic y Rodríguez 2005; Rodríguez et al. 1997) (Figura 13).

Las vasijas diaguita, en cambio, presentan diseños pintados de gran complejidad estructural: unidades mínimas como la greca escalerada utilizan varios principios simétricos conjuntamente, tales como reflexión desplazada, reflexión tipo espejo vertical y traslación (González 1997). Con ello se generan patrones ampliamente difundidos y estandarizados, los cuales han sido analizados por autores como Cornejo (1989) y González (1995). Por otra parte, la cuatripartición de campo de diseño está documentada por González en piezas de la zona, así como en diseños bidimensionales que son propios de la fase de aculturación incaica (González 1995, 2004) (Figura 13).

\section{Discusión y Conclusiones}

La tipología de figuras a partir de un marco nos permite visualizar la evolución de un diseño, que en términos formales va de un motivo figurativo que alude al rostro, a uno abstracto. $Y$ en términos estructurales, desde un campo compositivo donde los elementos se disponen bajo una simetría axial, a otros donde se combinan según movimientos simétricos de mayor complejidad.

Interpretamos esto como distintas etapas en una secuencia de confección de diseño, donde van cambiando las soluciones visuales y hay temas que van siendo superados por los creadores, en una continuidad que denota un mismo estilo (Gombrich 1997) (Figura 14).

El análisis de los paneles y el tipo de sitio en que se encuentran, nos muestra que esta secuencia tiene un punto de inflexión que la separa en dos modalidades que son congruentes con la presencia de dos grupos culturales diferentes (PAT y Diaguita) cuyos referentes materiales se relacionan por contigüidad espacial con los grabados. Asimismo, podemos asemejar la estructura decorativa cerámica PAT con las representaciones rupestres dentro de un marco de los tipos I, III y V; y la estrategia diaguita con los tipos VI y VII. Visión que apoyaría la tesis de dos estilos rupestres consecuentes con la expresión propia de cada una de estas poblaciones (PAT y Diaguita), como lo plantea Troncoso para Chalinga (en Becker 2003) y para otras zonas del Choapa (Troncoso 2004).

Sin embargo, coincidimos con Davis (1990) en que las relaciones entre entidades históricas y estilísticas no necesitan ser interpretadas como relaciones de expresión, sino como relaciones indicativas o sintomáticas, donde las descripciones estilísticas apuntan a una entidad histórica, pero no la proyectan y no están necesariamente causadas por ella.

Si partimos de la idea de que al menos una parte de la producción de petroglifos está ligada al Alfarero Temprano, como parece aceptar la mayoría de quienes trabajan el tema (Castillo 1985; Jackson et al. 2000; Mostny y Niemeyer 1983, por citar algunos), proponemos entonces la posibilidad que este estilo haya cambiado con la llegada de 

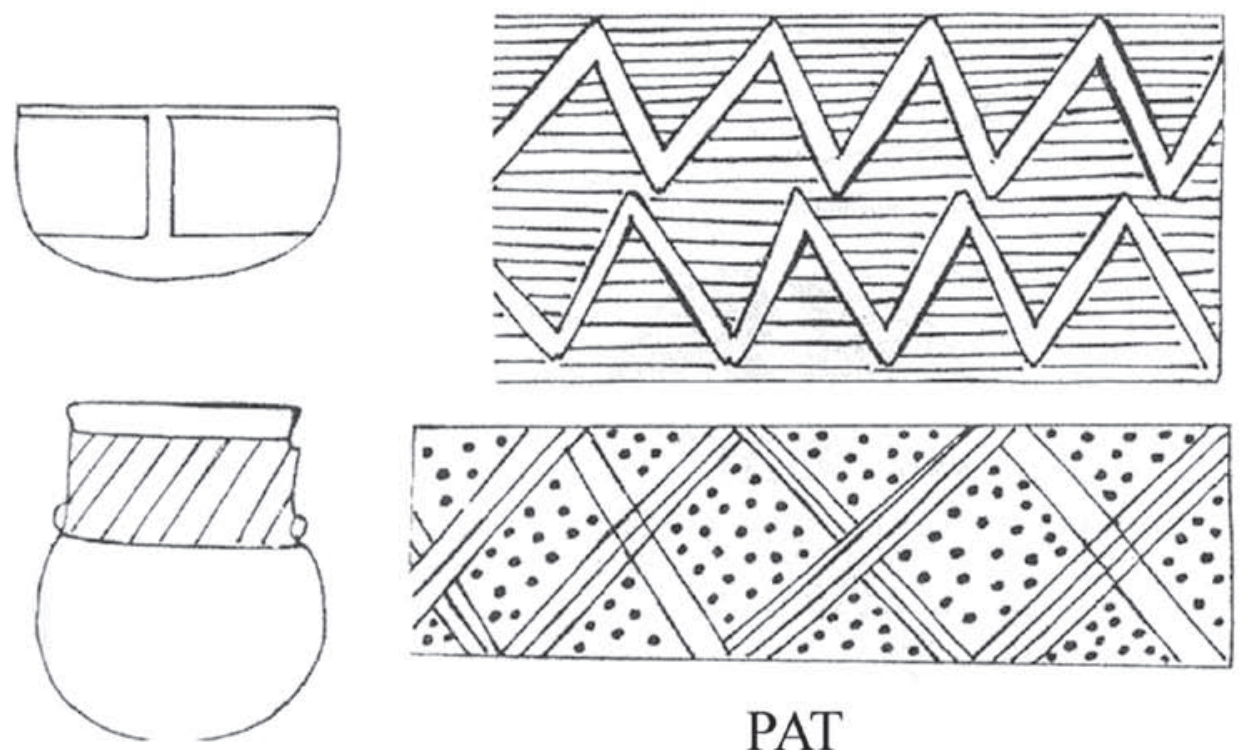

PAT
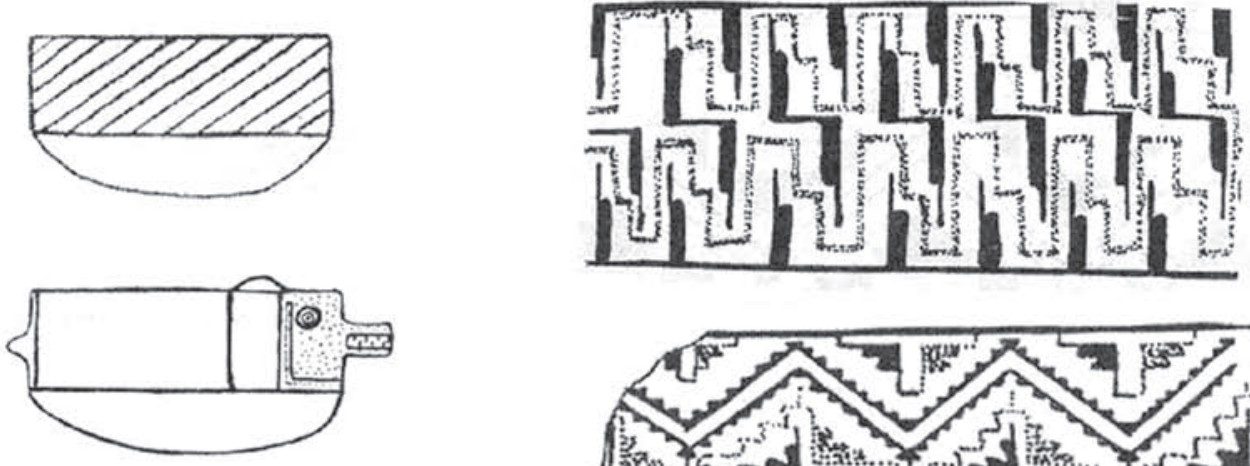

Diaguita


Figura 13. Otros referentes visuales utilizados en el análisis (tomado de González 1997). Other visual referents used in the analysis (after González 1997). 


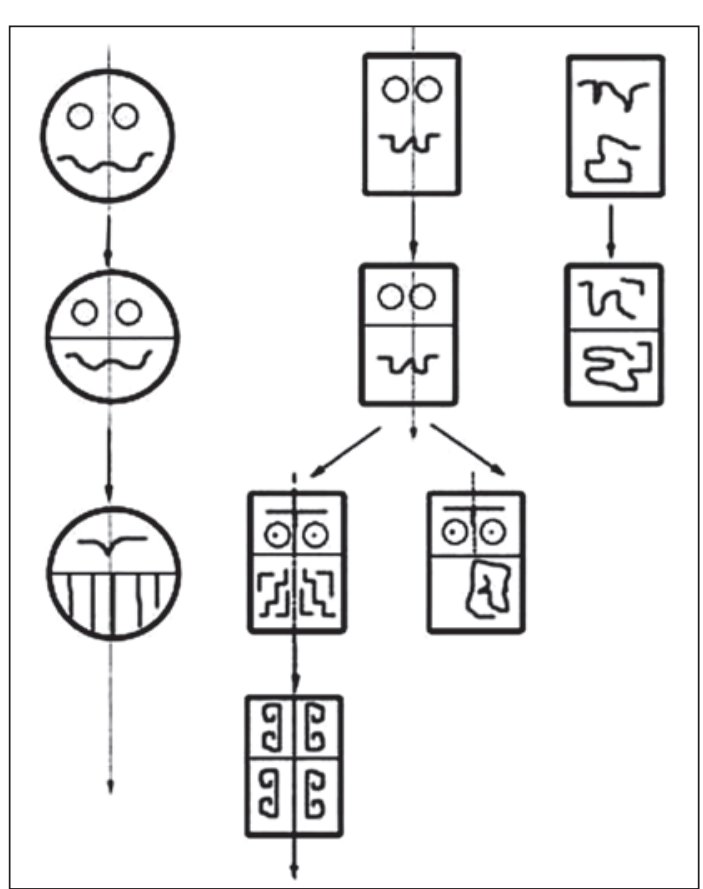

Figura 14. Esquema de la secuencia de diseño para las representaciones a partir de un marco.

Schemata of the design sequence for the framed figures.

poblaciones diaguita al área. Si consideramos que el arte es un medio de articulación social que está constantemente activo y ajustándose (Williams 1973), ya que es susceptible a los acontecimientos históricos y sociales de los cuales depende (Bourdieu 1967), el nuevo contexto impulsaría a los artistas locales a hacer innovaciones en el repertorio visual, incorporando nuevos motivos (geométricos y figurativos) y cambiando la configuración de las representaciones a partir de un marco, reproduciendo los diseños que se observan en las vasijas diaguita. Esto explicaría la falta de precisión en el uso de la simetría que muestran algunos de estos motivos, existiendo más bien una "intención simétrica" (sensu González 2005) que evidencia una apropiación a nivel de forma pero no de contenido.

En el nuevo escenario, las representaciones a partir de un marco dejarían su rol protagónico al combinarse con nuevos motivos de una forma más equivalente, pero sin desaparecer del repertorio visual. Esto ocurre en un nuevo tipo de sitio, de mayor visibilidad y de función distinta a la de vivienda, la mayoría bicomponente, lo cual podría estar reflejando no sólo un cambio en la estrategia visual de las poblaciones PAT sino lugares de interacción con los recién llegados.

Planteamos esta opción como una manera distinta de enfrentar el nuevo paisaje cultural de Chalinga, y del Choapa, donde parece cada vez más posible que poblaciones culturalmente diferentes estén compartiendo espacios en determinados momentos de la prehistoria.

Asimismo, esperamos dar cuenta de la complejidad del arte rupestre de Chalinga, desde una perspectiva que considera la dinámica y el cambio a través de la historia.

Somos conscientes de las limitaciones que enfrenta una tipología generada sobre un solo motivo, el cual -sin embargo- es crucial en el arte rupestre de la zona y en la definición de un estilo: el Limarí. Pero con ella se abren perspectivas y se cuestiona la pertinencia de dicho estilo, el cual pierde homogeneidad a medida que se definen similitudes y diferencias entre las representaciones rupestres de distintos sectores del Choapa (Artigas 2003; Castelleti 2004; Jackson et al. 2002; Troncoso 2001, 2004). De un modo similar a lo que ocurre desde la perspectiva cerámica, las diferencias valle a valle nos hablan de poblaciones que comparten un acervo cultural, pero que enfatizan ciertas cosas y minimizan otras, según intereses más bien locales. Para saber cuáles, se hace necesario y evidente seguir delineando cómo se comporta el arte rupestre de la zona.

Agradecimientos: A todos aquellos que colaboraron en mi Memoria, especialmente a Francisco Gallardo, Donald Jackson, Victoria Castro, Mauricio Uribe y Flora Vilches por las luces. A Diego Artigas, Cristian Becker, Paola González y Andrés Troncoso por la generosidad. A Juan Ignacio Cabello, Catalina Rivera y Francisca Gili por el don del arte. También a Leonid Velarde, Mauricio Lorca y a los evaluadores externos por sus valiosos comentarios al manuscrito. 


\section{Referencias Citadas}

Artigas, D.

2003 El Sueño Esculpido: Arte Rupestre y Memoria del Mito en el Valle de Canelillo. Provincia del Choapa. Memoria para optar al título de Arqueólogo, Departamento de Antropología, Universidad de Chile, Santiago.

Becker, C.

2003 Secuencia cronológica cultural y uso del espacio durante el período alfarero del valle de Chalinga, provincia del Choapa. Fondecyt $N^{\circ} 100039$. Manuscrito en posesión del autor.

Bourdieu, P.

1967 Campo intelectual y proyecto creador. En Problemas del Estructuralismo, editado por J. Pouillon, pp. 135-182. Siglo Veintiuno Editores S.A., México.

Cabello, G.

2003 Rostros que Hablan: Máscaras del valle de Chalinga. Actas VI Congreso Chileno de Antropología, Tomo II, pp. 1363-1370. Santiago.

2005 Rostros que Hablan: Una Propuesta Estilística para el Valle de Chalinga, IV Región. Memoria para optar al título de Arqueóloga, Departamento de Antropología, Universidad de Chile, Santiago.

Castelleti, J.

2004 El Arte Rupestre de Nocui en el Choapa. Memoria para optar al Título de Arqueólogo, Departamento de Antropología, Universidad de Chile, Santiago.

Castillo, G.

1985 Revisión del arte rupestre Molle. En Estudios en Arte Rupestre, editado por C. Aldunate, J. Berenguer y V. Castro, pp. 173-194. MCHAP, Santiago.

1990 Desarrollo prehispánico en la hoya hidrográfica del río Choapa. Manuscrito en posesión del autor.

Cornejo, L.

1989 El plato zoomorfo diaguita. Su variabilidad y especificidad. Boletín del Museo Chileno de Arte Precolombino 3:47-80.

Davis, W.

1990 Style and history in art history. En The Uses of Style in Archaeology, editado por M. Conkey y C. Hastorf, pp. 18-31. Cambridge University Press, Cambridge.

Gallardo, F.

1996 Acerca de la lógica en la interpretación del arte rupestre. Boletín Sociedad Chilena de Arqueología 23:31-33.

2009 Sobre la composición y la disposición en el arte rupestre de Chile: consideraciones metodológicas e interpretativas Magallania 37:85-98.

Gombrich, E.H.

1997 Enfoques de la historia del arte: tres puntos de discusión. En Gombrich Esencial, editado por Gombrich, E.H., pp. 355-367. Editorial Debate, Madrid.

González, P.

1995 Diseños Cerámicos Diaguita-Inka: Estructura, Simbolismo, Color y Relaciones Culturales. Memoria para optar al título de Arqueóloga, Departamento de Antropología, Universidad de Chile, Santiago.

1997 Patrones decorativos de las culturas Agroalfareras de la Provincia del Choapa y su relación con los patrones culturales de las áreas aledañas. Actas del XIV Congreso Nacional de Arqueología Chilena, Tomo 2, pp. 191-222. Copiapó.
2004 Estilo, interacción y poder: arte visual Diaguita Inca en asentamientos habitacionales del valle de Illapel y del área Diaguita nuclear. Werken 5:69-76.

2005 Códigos visuales de las pinturas rupestres Cueva Blanca: Formas, simetría y contexto. Boletín del Museo Chileno de Arte Precolombino 10(1):55-72.

Iribarren, J.

1973 Pictografías en las provincias de Atacama y Coquimbo. Boletín del Museo Arqueológico de La Serena 15:115132.

Jackson, D., D. Artigas y G. Cabello

2002 Trazos del Choapa. Arte Rupestre en la Cuenca del Río Choapa. Una Perspectiva Macroespacial. LOM Ediciones, Santiago.

Mostny, G. y H. Niemeyer

1983 Arte Rupestre Chileno. Publicación del Departamento de Extensión Cultural del Ministerio de Educación, Santiago.

Pavlovic, D.

2004 Dejando atrás la tierra de nadie: asentamientos, contextos y movilidad de las comunidades alfareras tempranas del Choapa. Werken 5:39-46.

Pavlovic, D. y J Rodríguez

2005 Nuevas proposiciones sobre el período alfarero temprano en la cuenca del Choapa. Actas del XVI Congreso Nacional de Arqueología Chilena, pp. 121-130. Tomé.

Rodríguez J., A. Troncoso, C. Becker, P. González y D. Pavlovic

1997 Ocupaciones prehispánicas en la cuenca del río Illapel. Actas del XIV Congreso Nacional de Arqueología Chilena, Tomo 2, pp. 331-344. Copiapó.

Sanhueza, L., D. Baudet, D. Jackson y L. Contreras 2004 La cultura Agrelo-Calingasta en el Choapa. Werken 5:47-52.

Schobinger, J.

1985 Relaciones entre los petroglifos del oeste de la Argentina y los de Chile. En Estudios en Arte Rupestre, editado por C. Aldunate, J. Berenguer y V. Castro, pp. 195-203. MCHAP, Santiago.

Troncoso, A.

2001 De monumentos y heterotopias: arte rupestre y paisaje en el curso superior del río Illapel, IV Región, Chile. Boletín del Museo Chileno de Arte Precolombino 8:9-20.

2004 Las posibilidades de la diferencia: una aproximación inicial al arte rupestre del valle del Choapa. Actas de Integrando la Arqueología del Choapa. Werken 5:127-132.

Urízar, G.

2004 El material cerámico del sitio Camisas 6 (Embalse Corrales), Comuna de Salamanca, Provincia del Choapa. Actas del XV Congreso Nacional de Arqueología Chilena. Chungara Revista de Antropología Chilena Vol. 36 Número Especial, Tomo 2, pp. 817-831.

Washburn D. y D. Crowe

1988 Theory and Practice of Plane Pattern Analysis. University of Washington Press, Seattle.

Williams, R.

1973 Base and superestructure in marxist cultural theory. New Left Review 1(82):3-16. 


\section{Notas}

1 Tendencia a la horizontalidad para el período Alfarero Temprano; oblicuo para el Intermedio Tardío (Diaguita); y la combinación de disposición vertical y horizontal, con la primacía ocasional de alguno de ellos, para el Tardío (Inca).

2 Este estudio, cuyos resultados se resumen en el presente artículo, se inserta en una investigación mayor del arte rupestre del Choapa: Proyecto de Investigación en Ciencias Sociales, Humanidades y Educación DID SOC.03-192. Universidad de Chile, investigador responsable: Donald Jackson.

3 Sobre este tema ver por ejemplo Pavlovic (2004); Sanhueza et al. (2004); Pavlovic y Rodríguez 2005.

4 Nos referimos particularmente a las campañas de prospección y registro realizadas para mi práctica profesional, en el marco de proyecto Fondecyt $n^{\circ} 1000039$, y a las de registro de los proyectos DID S00-12/2 y SOC.03-192. Para efectos prácticos, seguimos la nomenclatura de los sitios de este último, en el cual se enmarcó mi Memoria de Título.

5 El Tree Clustering usa las diferencias o distancias (Percent Disagreement) entre los objetos para formar grupos, a través del programa Stadistica 5.0.

6 Hemos incluido aquí tres casos en que si bien no está manifiesto el eje vertical, el diseño interior genera cuatro segmentos iguales, respondiendo a una estructura cuatripartita. Dos de ellos corresponden a los llamados "signos escudo", cuyo análisis simétrico representa una gran dificultad (comparable a la que representa el diseño cerámico conocido como "chevrón”, P. González com. pers. 2005), pero que Schobinger (1985) también postula como una esquematización de los mascariformes.

7 Gallardo (1996) señala que existe una lógica de facto en la interpretación del arte rupestre, que puede ser expresada por el uso de tres tipos de razonamiento asociativo: contraste, que intenta discernir patrones de organización a nivel de los diseños y sus asociaciones; semejanza, que relaciona similitudes formales entre motivos rupestres y artefactos (o motivos presentes en artefactos) de edad conocida; y la contigüidad del arte rupestre con las ocupaciones humanas del sitio, que enmarca cronológicamente la autoría entre la ocupación más temprana y la más tardía.

8 Los criterios que diferencian el tipo de sitio son: emplazamiento geográfico, dimensiones, cantidad y tipo de bloques, cantidad y tipo de motivos presentes en el panel. La adscripción cronocultural está limitada a la observación de la cerámica superficial, contando sólo con unos pocos pozos de sondeo y tres fechados TL.

9 Variables como el grado de patinación, técnicas de ejecución y la presencia de yuxtaposición, superposición o reactivación de los grabados fueron evaluadas sin ser factores indicativos en nuestro análisis, por lo que no se desarrollan en este artículo. Para más detalle ver Tabla 2 en Cabello (2005). 